\title{
Higher-order brane gravity models
}

\author{
Mariusz P. Dạbrowski and Adam Balcerzak \\ Institute of Physics, University of Szczecin, Wielkopolska 15, 70-451 Szczecin, Poland
}

\begin{abstract}
We discuss a very general theory of gravity, of which Lagrangian is an arbitrary function of the curvature invariants, on the brane. In general, the formulation of the junction conditions (except for Euler characteristics such as Gauss-Bonnet term) leads to the powers of the delta function and requires regularization. We suggest the way to avoid such a problem by imposing the metric and its first derivative to be regular at the brane, the second derivative to have a kink, the third derivative of the metric to have a step function discontinuity, and no sooner as the fourth derivative of the metric to give the delta function contribution to the field equations. Alternatively, we discuss the reduction of the fourth-order gravity to the second order theory by introducing extra scalar and tensor fields: the scalaron and the tensoron. In order to obtain junction conditions we apply two methods: the application of the Gauss-Codazzi formalism and the application of the generalized Gibbons-Hawking boundary terms which are appended to the appropriate actions. In the most general case we derive junction conditions without assuming the continuity of the scalaron and the tensoron on the brane. The derived junction conditions can serve studying the cosmological implications of the higher-order brane gravity models.
\end{abstract}

Keywords: higher-order gravities, braneworld, junction conditions, boundary terms

PACS: $04.50 . \mathrm{Kd} ; 11.25 .-\mathrm{w}$; 98.80.-k; 98.80.Jk

\section{INTRODUCTION}

In recent years there have been growing interest both in brane universes [1, 2] and in the higher-order gravity theories of which the simplest is $f(R)$ gravity [3]. In this talk we are going to combine both ideas and formulate the higher-order gravities on the brane. It emerges that the formulation is a bit non-trivial, since one faces ambiguities of the quadratic delta function contributions to the field equations. We will say how to avoid these problems and show how the Israel junction conditions for such higher-order brane gravity models can be formulated.

\section{FOURTH-ORDER GRAVITIES.}

When one considers the general gravity theories (e.g. [4, 5]):

$$
S=\chi^{-1} \int d^{D} \sqrt{-g} f(X, Y, Z)
$$

in a D-dimensional spacetime ( $\chi=$ const.), where $X, Y, Z$ are curvature invariants

$$
X=R, \quad Y=R_{a b} R^{a b}, \quad Z=R_{a b c d} R^{a b c d},
$$

then one immediately faces the 4 th order field equations, except when they reduce to the theories with Euler densities of the n-th order $I^{(n)}$ [6]

$$
S=\int_{M} d^{D} x \sqrt{-g} \sum_{n} \kappa_{n} I^{(n)}
$$

the lowest of them being the cosmological constant $I^{(0)}=1\left(\kappa_{0}=-2 \Lambda\left(2 \kappa^{2}\right)^{-1}=-2 \Lambda / 16 \pi G\right)$, the Ricci scalar $I^{(1)}=R\left(\kappa_{1}=\left(2 \kappa^{2}\right)^{-1}\right)$, and the Gauss-Bonnet density $I^{(2)}=R G B=R^{2}-4 R_{a b} R^{a b}+R_{a b c d} R^{a b c d}\left(\kappa_{2}=\alpha\left(2 \kappa^{2}\right)^{-1}\right.$, $\alpha=$ const.).

However, the theories based on the Lagrangians which are the functions of the Euler densities such as

$$
f(R)=f(X), \quad f\left(R_{G B}\right)=f\left(Z-4 Y+X^{2}\right), \quad f=f\left(I^{(n)}\right)
$$

are again fourth-order. 


\section{FORMULATION OF THE 4TH ORDER GRAVITIES ON THE BRANE - ISRAEL FORMALISM.}

In the context of the recent interest in string/M-theory, it is interesting to formulate the general gravity theories (1) within the framework of the brane models [7]. The full brane action for such a theory reads as

$$
S=\chi^{-1} \int_{M} d^{D} x \sqrt{-g} f(X, Y, Z)+S_{\text {brane }}+S_{m}
$$

with the total energy-momentum tensor

$$
T_{a}^{b}=T_{a}^{b-} \boldsymbol{\theta}(-w)+T_{a}^{b+} \boldsymbol{\theta}(w)+\delta(w) S_{a}^{b}
$$

where $S_{a}^{b}$ is the energy-momentum tensor on the brane, and $T_{a}{ }^{b}$ are the energy-momentum tensors on the both sides of the brane, $\theta(w)$ is the Heaviside step function, and $\delta(w)$ is the Dirac delta function.

We assume Gaussian normal coordinates, i.e., $(\mu, v=0,1,2, \ldots, D-2 ; w=D)$

$$
d s^{2}=g_{a b} d x^{a} d x^{b}=\varepsilon d w^{2}+h_{\mu v} d x^{\mu} d x^{v}
$$

where $\varepsilon=\vec{n} \cdot \vec{n}=+1$ for a spacelike hypersurface, $\varepsilon=-1$ for a timelike hypersurface, and $h_{a b}=g_{a b}-\varepsilon n_{a} n_{b}$ is a projection tensor onto a $(D-1)$-dimensional hypersurface, $\vec{n}$ is the normal vector to the hypersurface. In these coordinates the extrinsic curvature is

$$
K_{\mu v}=-\frac{1}{2} \frac{\partial h_{\mu v}}{\partial w}
$$

and the Gauss-Codazzi equations read [8]

$$
\begin{aligned}
R_{w \mu w v} & =\frac{\partial K_{\mu v}}{\partial w}+K_{\rho v} K_{\mu}^{\rho} \\
R_{w \mu v \rho} & =\nabla_{v} K_{\mu \rho}-\nabla_{\rho} K_{\mu v} \\
R_{\lambda \mu v \rho} & ={ }^{(D-1)} R_{\lambda \mu v \rho}+\varepsilon\left[K_{\mu v} K_{\lambda \rho}-K_{\mu \rho} K_{\lambda v}\right] .
\end{aligned}
$$

In the standard Israel approach [9] one assumes that at the brane position $w=0$ :

$$
\begin{aligned}
h_{\mu v}^{-} & =h_{\mu v}^{+} \\
h_{\mu v, w}^{-} & \neq h_{\mu v, w}^{+}, \quad K_{\mu v}^{-} \neq K_{\mu v}^{+}
\end{aligned}
$$

i.e., the metric is continuous but it has a kink, its first derivative has a step function discontinuity, and its second derivative gives the delta function contribution.

In terms of $\theta(w)$ and $\delta(w)$ functions this is equivalent to

$$
\begin{aligned}
h_{\mu v}(w) & =h_{\mu v}^{-}(w) \theta(-w)+h_{\mu v}^{+}(w) \theta(w) \\
\frac{\partial h_{\mu v}}{\partial w} & =\frac{\partial h_{\mu v}^{+}}{\partial w} \theta(-w)+\frac{\partial h_{\mu v}^{-}}{\partial w} \theta(w) \\
\frac{\partial^{2} h_{\mu v}}{\partial w^{2}} & =\frac{\partial^{2} h_{\mu v}^{-}}{\partial w^{2}} \theta(-w)+\frac{\partial^{2} h_{\mu v}^{+}}{\partial w^{2}} \theta(w) \\
& +\left(\frac{\partial h_{\mu v}^{-}}{\partial w}-\frac{\partial h_{\mu v}^{+}}{\partial w}\right) \delta(w)
\end{aligned}
$$

For the standard brane models with the Einstein-Hilbert action in the bulk

$$
S=\frac{1}{2 \kappa^{2}} \int_{M} d^{D} x \sqrt{-g} R+S_{\text {brane }}+S_{m}
$$


the field equations read as [8]

$$
\begin{aligned}
G_{w}^{w} & =-\frac{1}{2}{ }^{(D-1)} R+\frac{1}{2} \varepsilon\left[K^{2}-\operatorname{Tr}\left(K^{2}\right)\right]=\kappa^{2} T_{w}^{w}, \\
G_{\mu}^{w} & =\varepsilon\left[\nabla_{\mu} K-\nabla_{v} K_{\mu}^{v}\right]=\kappa^{2} T_{\mu}^{w} \\
G_{v}^{\mu} & ={ }^{(D-1)} G_{v}^{\mu}+\varepsilon\left[\frac{\partial K_{v}^{\mu}}{\partial w}-\delta_{v}^{\mu} \frac{\partial K}{\partial w}\right] \\
& +\varepsilon\left[-K K_{v}^{\mu}+\frac{1}{2} \delta_{v}^{\mu} \operatorname{Tr}\left(K^{2}\right)+\frac{1}{2} \delta_{v}^{\mu} K^{2}\right]=\kappa^{2} T_{v}^{\mu} .
\end{aligned}
$$

and in the $\operatorname{limit}_{\lim } \rightarrow 0 \int_{-w}^{w}$, which "fishes out" the delta function contributions, one gets the standard Israel junction conditions as [8]:

$$
\varepsilon\left\{\left[K_{v}^{\mu}\right]-\delta_{v}^{\mu}[K]\right\}=\kappa^{2} S^{\mu}, \quad\left[K_{v}^{\mu}\right] \equiv K_{v}^{\mu+}-K_{v}^{\mu-} .
$$

By $[X]=X^{+}+X^{-}$we define a jump of an appropriate quantity $X$ at the brane.

However, for the general $f(X, Y, Z)$ theory on the brane, the standard continuity relations $(12)-(13)$ do not work. This can be seen from the field equations of the action (1)

$$
\begin{aligned}
P_{a b} & =\frac{\chi}{2} T_{a b}, \\
P^{a b} & =-\frac{1}{2} f g^{a b}+f_{X} R^{a b}+2 f_{Y} R^{c(a} R_{c}^{b)}+2 f_{Z} R^{e d c(a} R^{b)} c d e \\
& +f_{X ; c d}\left(g^{a b} g^{c d}-g^{a c} g^{b d}\right)+\square\left(f_{Y} R^{a b}\right)+g^{a b}\left(f_{Y} R^{c d}\right)_{; c d} \\
& -2\left(f_{Y} R^{c(a}\right)_{; c}^{b)}-4\left(f_{Z} R^{d(a b) c}\right)_{; c d},
\end{aligned}
$$

where $f_{X}=\partial f / \partial X$ etc.

Take, for example, the square of the Ricci scalar

$$
R={ }^{(D-1)} R+\varepsilon\left[2 h^{\mu v} \frac{\partial K_{\mu v}}{\partial w}+3 \operatorname{Tr}\left(K^{2}\right)-K^{2}\right],
$$

where $K \equiv K_{\mu}^{\mu}, \operatorname{Tr}\left(K^{2}\right) \equiv K^{\mu v} K_{\mu v}$, and appropriately, of the Ricci tensor, and of the Riemann tensor. These squares produce the terms of the type

$$
\frac{\partial^{2} h^{\mu v}}{\partial^{2} w} \frac{\partial K_{\mu v}}{\partial w}, \frac{\partial K_{\mu v}}{\partial w} \frac{\partial K^{\mu v}}{\partial w},\left(\frac{\partial K}{\partial w}\right)^{2}
$$

which are proportional to $\delta^{2}(w)$, and so they are ambiguous.

Amazingly, all these ambiguous terms cancel each other exactly in the case of the Euler densities [10]. In fact, the junction conditions for one of the Euler densities - the Gauss-Bonnet density, were already obtained as [11, 21]

$$
2 \alpha\left(3\left[J_{\mu v}\right]-[J] h_{\mu v}-2[P]_{\mu \rho v \sigma}[K]^{\rho \sigma}\right)+\left[K_{\mu v}\right]-[K] h_{\mu v}=-\kappa^{2} S_{\mu v}
$$

where

$$
\begin{aligned}
P_{\mu \rho v \sigma} & =R_{\mu \rho v \sigma}+2 h_{\mu[\sigma} R_{v] \rho}+2 h_{\rho[v} R_{\sigma] \mu}+R h_{\mu[v} h_{\sigma] \rho} \\
J_{\mu v} & =\frac{1}{3}\left(2 K K_{\mu \sigma} K_{v}^{\sigma}+K_{\sigma \rho} K^{\sigma \rho} K_{\mu v}-2 K_{\mu \rho} K^{\rho \sigma} K_{\sigma v}-K^{2} K_{\mu v}\right) .
\end{aligned}
$$

In the limit $\alpha \rightarrow 0$, they just give Einstein-Hilbert action junction conditions 21 .

In view of the ambiguities of the terms in (24), we find two ways to formulate the junction conditions for general $f(X, Y, Z)$ theories on the brane. 


\section{A. Smoothing out the continuity conditions for the metric tensor at the brane}

In order to do that we impose more regularity onto the metric tensor at the brane position, i.e., we consider $a$ singular hypersurface of the order three [9] which fulfills the conditions (compare (12)-(13))

$$
\begin{aligned}
h_{\mu v}^{-} & =h_{\mu v}^{+}, \\
h_{\mu v, w}^{-} & =h_{\mu v, w}^{+}, \quad K_{\mu v}^{-}=K_{\mu v}^{+}, \\
h_{\mu v, w w}^{-} & =h_{\mu v, w w}^{-}, \quad K_{\mu v, w}^{-}=K_{\mu v, w}^{+}, \\
h_{\mu v, w w w}^{-} & \neq h_{\mu v, w w w}^{+}, \quad K_{\mu v, w w}^{-} \neq K_{\mu v, w w}^{+},
\end{aligned}
$$

i.e., the metric and its first derivative are regular, the second derivative of the metric is continuous, but possesses a kink, the third derivative of the metric has a step function discontinuity, and no sooner than the fourth derivative of the metric on the brane produces the delta function contribution.

The physical interpretation as put in terms of the second-order theory can be that there is a jump of the first derivative of the energy-momentum tensor (e.g. jump of a pressure gradient) at the brane.

In his seminal work, Israel [9] proposed a singular hypersurface of order two, which physically corresponded to a boundary surface characterized by a jump of the energy-momentum tensor (e.g. a boundary surface separating a star from the surrounding vacuum) which was characterized by

$$
\begin{aligned}
h_{\mu v}^{-} & =h_{\mu v}^{+}, \\
h_{\mu v, w}^{-} & =h_{\mu v, w}^{+}, \quad K_{\mu v}^{-}=K_{\mu v}^{+}, \\
h_{\mu v, w w}^{-} & \neq h_{\mu v, w w}^{-}, \quad K_{\mu v, w}^{-}=K_{\mu v, w}^{+},
\end{aligned}
$$

i.e., the metric is regular, the first derivative of the metric is continuous, but possesses a kink, the second derivative of the metric has a step function discontinuity, and the third derivative of the metric on the brane produces the delta function contribution.

The appropriate junction conditions can be obtained as follows. We rewrite the field equations 22, 23 as

$$
\sqrt{-g} C_{a b} W_{; d}^{a b d}+\sqrt{-g} C_{a b} V^{a b}=\frac{\chi}{2} T^{a b} C_{a b} \sqrt{-g},
$$

where we have introduced is an arbitrary tensor field $C_{a b}$, and

$$
\begin{aligned}
W^{a b d} & =f_{X ; c}\left(g^{a b} g^{c d}-g^{(a c} g^{b) d}\right)+\left(f_{Y} R^{a b}\right)^{; d} \\
& +g^{a b}\left(f_{Y} R^{c d}\right)_{; c}-2\left(f_{Y} R^{d(a)}\right)^{; b)}-4\left(f_{Z} R^{d(a b) c}\right)_{; c} \\
V^{a b} & =-\frac{1}{2} f g^{a b}+f_{X} R^{a b}+2 f_{Y} R^{c(a} R^{b)}{ }_{c} \\
& +2 f_{Z} R^{e d c(a} R^{b)}{ }_{c d e},
\end{aligned}
$$

contain third derivatives of the metric giving a step function discontinuity, so that $W^{a b d}$ is proportional to $\delta(w)$. Then, we integrate both sides of the formula (35) over the volume $V$ which contains the following parts (cf. Fig. 1): $G 1, G 2$ - are the left-hand-side and the right-hand-side bulk volumes which are separated by the brane, $A 1=\partial G 1+A 0$, $A 2=\partial G 2-A 0$ are the boundaries of these volumes, and $A 0$ is the brane which orientation is given by the direction of the normal vector $\vec{n}$.

We have

$$
\int_{G 1+G 2} \sqrt{-g} C_{a b} W_{; d}^{a b d} d \Omega+\int_{G 1+G 2} \sqrt{-g} C_{a b} V^{a b} d \Omega=\int_{G 1+G 2} \frac{\chi}{2} T^{a b} C_{a b} \sqrt{-g} d \Omega,
$$

and so

$$
\begin{aligned}
& \int_{G 1+G 2} \sqrt{-g}\left(C_{a b} W^{a b d}\right)_{; d} d \Omega-\int_{G 1+G 2} \sqrt{-g} C_{a b ; d} W^{a b d} d \Omega+\int_{G 1+G 2} \sqrt{-g} C_{a b} V^{a b} d \Omega \\
= & \int_{G 1} \frac{\chi}{2} T^{a b} C_{a b} \sqrt{-g} d \Omega+\int_{G 2} \frac{\chi}{2} T^{a b} C_{a b} \sqrt{-g} d \Omega+\int_{A 0} \frac{\chi}{2} S^{a b} C_{a b} \sqrt{-\gamma} d \sigma,
\end{aligned}
$$




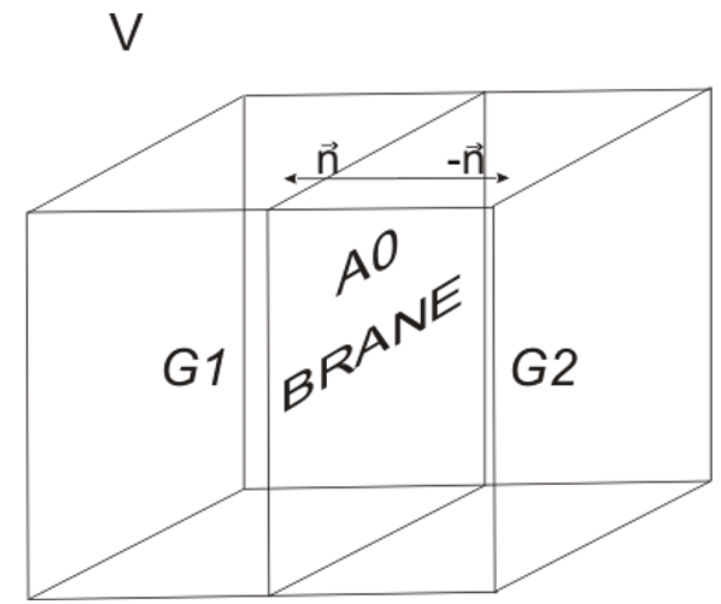

FIGURE 1. A schematic picture illustrating the domains of integration used in derivation of the junction conditions. Here $V=G 1+G 2$ is the total volume, $G 1, G 2$ - are the left-hand-side and the right-hand-side bulk volumes which are separated by the brane, $A 1=\partial G 1+A 0, A 2=\partial G 2-A 0$ are the boundaries of these volumes, and $A 0$ is the brane which orientation is given by the direction of the normal vector $\vec{n}$.

of which the first term can be integrated out to a boundary A1+A2 and then the limit $V \rightarrow A 0$ (or $\lim _{w \rightarrow 0} \int_{-w}^{w}$ in Gaussian coordinates) is taken.

The final form of the junction conditions which generalize 21] onto the fourth-order gravity are

$$
[W]^{a b d} n_{d}-\frac{\chi}{2} S^{a b}=0, \quad[W]^{a b d}=W^{a b d+}-W^{a b d-} .
$$

It is remarkable that these junction conditions involve the higher derivatives of the scale factor. To see this take for example $f(X, Y, Z)=f(R)$ theory in $D=5$ dimensions with metric

$$
d s^{2}=-d t^{2}+a^{2}(t, w)\left[d r^{2}+r^{2}\left(d \Theta^{2}+\sin ^{2} \Theta d \phi^{2}\right)\right]+d w^{2} .
$$

The junction conditions 40 give a jump of the third derivative of $a(t, w)$, as expected

$$
\begin{aligned}
{\left[a^{\prime \prime \prime}\right] } & =\frac{\chi}{2} a_{0} p_{0}, \\
p_{0} & =\rho_{0},
\end{aligned}
$$

where $(\ldots)^{\prime}=\partial / \partial w, a_{0}=a(w=0)$, and the brane energy-momentum tensor is $S_{\mu}^{\nu}=\left(-\rho_{0}, p_{0}, p_{0}, p_{0}\right)$.

\section{B. Reduction to an equivalent 2nd order theory}

Yet another way to obtain the junction conditions is the reduction of the action (11) to a second-order action. This gives equivalent junction conditions, though at the expense of introducing a new tensor field $H^{a b c d}$ (tensoron). In fact, starting from the action [12]

$$
S_{G}=\chi^{-1} \int_{M} d^{D} x \sqrt{-g} f\left(g_{a b}, R_{a b c d}\right) .
$$

we may transform to an equivalent 2nd order action in the form

$$
S_{I}=\chi^{-1} \int_{M} d^{D} x \sqrt{-g}\left\{H^{g h i j}\left(R_{g h i j}-\phi_{g h i j}\right)+f\left(g_{a b}, \phi_{c d e f}\right)\right\},
$$


where

$$
H^{g h i j} \equiv \frac{\partial f\left(g_{a b}, \phi_{a b c d}\right)}{\partial \phi_{g h i j}}, \quad \operatorname{det}\left[\frac{\partial^{2} f\left(g_{a b}, \phi_{a b c d}\right)}{\partial \phi_{g h i j} \partial \phi_{k l m n}}\right] \neq 0 .
$$

This transition for $f(R)$ theory requires a new scalar $H=f^{\prime}(Q)$ (a scalaron) with the condition that $f^{\prime \prime}(Q) \neq 0$, and the equation of motion $Q=R$. Similarly, for $f\left(R_{G B}\right)$ theory, one defines a scalar $H=f^{\prime}(A)$, with the equation of motion $\left.A=R_{G B}\right)$. In order to get junction conditions, we have to slightly redefine the tensoron

$$
A^{a b c d}=\frac{1}{2}\left\{H^{a c d b}+H^{a b d c}-H^{c b d a}-H^{a c b d}-H^{a b c d}+H^{c b a d}\right\}
$$

which in a particular case of $f(X, Y, Z)$ theory takes the form

$$
A^{a b c d}=f_{X}\left(g^{a d} g^{c b}-g^{c d} g^{b a}\right)+f_{Y}\left(2 R^{a d} g^{b c}-R^{c d} g^{b a}-R^{b a} g^{c d}\right)+4 f_{Z} R^{a c b d} .
$$

The field equations for an equivalent action (43) read as

$$
\begin{aligned}
R_{g h i j} & =-\frac{\partial V\left(g_{a b}, H^{c d e f}\right)}{\partial H^{g h i j}} \\
\frac{1}{2} g^{a b} f & +\frac{\partial f}{\partial g_{a b}}+H^{b e c d} \phi_{e c d}^{a}\left(g_{a b}, H^{k l m n}\right)+\left\{A^{(a b) c d}\right\}_{; d c}=-\frac{\chi}{2} T^{a b}
\end{aligned}
$$

where

$$
V\left(g_{a b}, H^{c d e f}\right)=-H^{h g i j} \phi_{g h i j}\left(g_{a b}, H^{c d e f}\right)+f\left(g_{a b}, \phi_{k l m n}\left(g_{a b}, H^{c d e f}\right)\right)
$$

In fact, the possibility to express the fields $\phi_{a b c d}$ as a function of $g_{a b}$ and $H^{c d e f}$ is guaranteed by the condition 45 (which is an analogue of the condition $f^{\prime \prime}(Q) \neq 0$ ).

One can show that junction conditions of the second-order theory are equivalent to junction conditions of the fourthorder theory [7].

Applying the same method as in the previous case (i.e. taking the limit of $V \rightarrow A 0$ ) we notice that the first three terms of (49) do not give any contribution to the junction conditions (since they do not contain delta functions at all) which now have the form:

$$
\left[A_{; d}^{(a b) c d}\right] n_{c}=-\frac{\chi}{2} S^{a b}
$$

Assuming that

$$
f\left(g_{a b}, \phi_{a b c d}\right)=f\left(\phi_{a b}^{a b}, \phi_{a c b}{ }^{c} \phi^{a c b}{ }_{c}, \phi_{a b c d} \phi^{a b c d}\right),
$$

we can get the same result as in the 4th theory

$$
\begin{aligned}
{\left[A_{; d}^{(a b) c d}\right] n_{c} } & =\left[A_{; c}^{(a b) c d}\right] n_{d}=\left[-\left\{f_{X ; c}\left(g^{a b} g^{c d}-g^{c(a} g^{b) d}\right)\right.\right. \\
& +\left(f_{Y} R^{a b}\right)^{; d}+g^{a b}\left(f_{Y} R^{c d}\right)_{; c} \\
-2\left(f_{Y} R^{d(a)} ; b\right) & \left.\left.-4\left(f_{Z} R^{d(a b) c}\right)_{; c}\right\}\right] n_{d}=-\left[W^{a b d}\right] n_{d} .
\end{aligned}
$$

Similar approach was used for less-general $f(R)$ theories of gravity on the brane by Borzeszkowski and Frolov [13]; Parry at al. [14], Deruelle et al. [15], and for $f(X, Y, Z)=a X^{2}+b Y+c Z(a, b, c=$ const.) theories by Nojiri and Odintsov [5]. 


\section{FORMULATION OF THE 4TH ORDER GRAVITIES ON THE BRANE - GIBBONS-HAWKING BOUNDARY TERMS}

In this approach, following the idea of Gibbons and Hawking [16], we do not assume any vanishing of the first derivative of the variation of the metric tensor $\delta g_{a b ; c}$ on the boundary of the integration volume while using the variational principle. Strictly speaking, only the assumption of the vanishing of the normal derivative of the variation of the metric tensor $\delta g_{a b, w}$ is required. Instead, we postulate that some extra terms to the action are added and that these terms "kill" the first derivatives of the metric variation. These terms are called Gibbons-Hawking boundary terms now. In fact, the Gibbons-Hawking boundary term for the Einstein-Hilbert action is composed of the trace of the extrinsic curvature and it was found by Gibbons and Hawking themselves [16]. Then, for the action being the combination of the square of the Weyl tensor and an arbitrary function of the scalar curvature they were found by Hawking and Lutrell [17] and Barrow and Madsen [18]. For the Gauss-Bonnet and other Lovelock densities they were found by Bunch [19], Mueller-Hoissen and Myers [20], Davis [21] and Gravanis and Willinson [22]. The boundary terms for the action being an arbitrary function of the curvature invariants were found by Barvinsky and Solodukhin [23].

For the theories which are of interest for this talk, the Gibbons-Hawking boundary terms have the following form [24]: for the $f(R)$ theory the term reads as

$$
S_{G H, p}=-2(-1)^{p} \varepsilon \int_{\partial M_{p}} \sqrt{-h} H K d^{D-1} x,
$$

where $H=f^{\prime}(Q)$ is the scalaron, while for the $f(X, Y, Z)$ theory it reads as

$$
S_{G H, p}=-(-1)^{p} \int_{\partial M_{p}} d^{D-1} x \sqrt{-h} A^{(a b) c d} n_{c} n_{d} \mathscr{L}_{\vec{n}} g_{a b}
$$

where $A^{(a b) c d}$ is the tensoron. [24]:

Using the method of the boundary terms we derived the most general Israel junction conditions for $f(R)$ theory as

$$
\begin{aligned}
{[K] } & =0, \\
S^{a b} n_{a} n_{b} & =0, \\
S^{a b} h_{a c} n_{b} & =0, \\
-(D-1)\left[H_{; c} n^{c}\right]-D[H] K & =\varepsilon \frac{\chi}{2} S^{a b} h_{a b}, \\
-h_{a b}\left[H_{; c} n^{c}\right]-[H] K h_{a b} & +\left[H K_{a b}\right] \\
& =\varepsilon \frac{\chi}{2} S^{c d} h_{c a} h_{d b} .
\end{aligned}
$$

A generality of these conditions refers to the fact that no assumption about the continuity of the scalaron on the brane has been made. They reduce to the conditions already obtained in the literature, if one assumes $[H]=0[15]$.

On the other hand, the most general Israel junction conditions for the $f(X, Y, Z)$ theory, with no assumption about the continuity of the tensoron on the brane, are [24]:

$$
\begin{aligned}
& {\left[K A^{(a b) c d}\right] n_{c} n_{d}+\left[\mathscr{L}_{\vec{n}} A^{(a b) c d}\right] n_{c} n_{d} } \\
- & \varepsilon\left[A^{(a b) c d} K_{c d}\right]-g^{a b}\left[A^{(e f) c d} K_{e f}\right] n_{c} n_{d} \\
+ & 2 \varepsilon\left[D_{s} A^{(e f) c d} n_{c} n_{d}\right] h_{e}^{s} h_{f}^{(a} n^{b)}-2 \varepsilon\left[A_{;(c) c d}^{(a b) c}\right] n_{d)}=\frac{\chi}{2} S^{a b}, \\
& n_{b} n_{c}\left[\mathscr{L}_{\vec{n}} g_{a d}\right]-n_{a} n_{c}\left[\mathscr{L}_{\vec{n}} g_{d b}\right]-n_{b} n_{d}\left[\mathscr{L}_{\vec{n}} g_{a c}\right]+n_{a} n_{d}\left[\mathscr{L}_{\vec{n}} g_{c b}\right]=0 .
\end{aligned}
$$

They reduce to the conditions 51 , if one assumes continuity of the tensoron on the brane

$$
\left[A^{(a b) c d}\right]=0 .
$$




\section{FOURTH-ORDER GRAVITIES AND STATEFINDERS}

We claim the fact that general $f\left(R, R_{a b} R^{a b}, R_{a b c d} R^{a b c d}\right)$ theories are fourth-order may have some advantageous consequences onto their observational verification by the application of statefinder diagnosis of the universe.

In fact, statefinders are the higher-order characteristics of the universe expansion which go beyond the Hubble parameter $H$ and the deceleration parameter $q$ :

$$
H=\frac{\dot{a}}{a}, \quad q=-\frac{1}{H^{2}} \frac{\ddot{a}}{a}=-\frac{\ddot{a} a}{\dot{a}^{2}} .
$$

They can generally be expressed as $(i \geq 2)$

$$
x^{(i)}=(-1)^{i+1} \frac{1}{H^{i}} \frac{a^{(i)}}{a}=(-1)^{i+1} \frac{a^{(i)} a^{i-1}}{\dot{a}^{i}},
$$

and the lowest order of them are known as: jerk, snap ("kerk"), crack ("lerk")

$$
j=\frac{1}{H^{3}} \frac{\dot{\ddot{a}}}{a}=\frac{\dot{\ddot{a}} a^{2}}{\dot{a}^{3}}, \quad k=-\frac{1}{H^{4}} \frac{\ddot{a}}{a}=-\frac{\ddot{a} a^{3}}{\dot{a}^{4}}, l=\frac{1}{H^{5}} \frac{a^{(5)}}{a}=\frac{a^{(5)} a^{4}}{\dot{a}^{5}},
$$

and pop ("merk"), "nerk", "oerk", "perk" etc. [25].

In the case of the 4th order gravities, statefinders may become powerful tools to constrain such theories observationally, since they enter observational relations in the higher orders of redshift $z$ (see [27] for non-brane case diagnosis).

Apparently, a blow-up of statefinders may also be linked to an emergence of exotic singularities in the universe [26].

\section{CONCLUSIONS}

We conclude the following:

- The formulation of the fourth-order gravity theories on the brane is non-trivial because of the powers of delta function ambiguities.

- Two methods were applied:

A. Smoothing out the continuity conditions for the metric tensor at the brane;

B. Reduction to an equivalent 2nd order theory.

In both cases the Israel junction conditions have been obtained and they are also mutually equivalent.

- The method of the GH boundary terms was also applied and the most general junction conditions (with no continuity of the scalaron and tensoron on the brane assumed) were obtained that way, too.

- Higher-order brane gravities contain higher-order derivatives of the geometric quantities (in a Friedmann model it is just the scale factor) which may manifest themselves in the higher-order characteristics of expansion such as statefinders (jerk, snap, lerk/crack, merk/pop).

- A blow-up of statefinders may be linked to an emergence of exotic singularities in the universe.

\section{ACKNOWLEDGMENTS}

We acknowledge the support of the Polish Ministry of Science and Higher Education grant No N N202 191234 (years 2008-10).

\section{REFERENCES}

1. L. Randall and R. Sundrum, Phys. Rev. Lett., 83, 3370 (1999); L. Randall and R. Sundrum, ibidem, 83, 4690 (1999).

2. M. Visser, Phys. Lett. B159, 22 (1985); N. Arkani-Hamed, S. Dimopoulos, and G. Dvali, Phys. Lett. B 516, 70 (1998); I.

Antoniadis, N. Arkani-Hamed, S. Dimopoulos, G. Dvali, Phys. Lett. B 436, 257 (1998); N. Arkani-Hamed, S. Dimopoulos, and G. Dvali, Phys. Rev. D 59, 086004 (1999). 
3. A.A. Starobinsky, Phys. Lett. B 91, 99 (1980); G. Magnano and L.M. Sokołowski, Phys. Rev. D 50, 5039 (1994); T.P. Sotiriou, Class. Quantum Grav. 23, 5117 (2006); G.J. Olmo, Phys. Rev. Lett. 98, 061101 (2007); G.J. Olmo, Phys. Rev. D 75, 023511 (2007); S. Capozziello, V.F. Cardone, and A. Troisi, Phys. Rev. D 71, 043503 (2005); S. Capozziello, S. Nojiri, S.D. Odintsov, and A. Troisi, Phys. Lett. B 639, 135 (2006); T. Chiba, T. Kobayashi, M. Yamaguchi, and J. Yokoyama, Phys. Rev. D 75, 043516 (2007); S. Capozziello and R. Garatini, Class. Quantum Grav. 24, 1627 (2007); L. Amendola, D. Polarski, and S. Tsujikawa, Phys. Rev. Lett. 98, 131302 (2007).

4. T. Clifton and J.D. Barrow, Phys. Rev. D72, 123003 (2005); Class. Quantum Grav. 23, 2951 (2006).

5. S. Nojiri and S.D. Odintsov, JHEP 007, 049 (2000); S. Nojiri, S.D. Odintsov and S. Ogushi, Phys. Rev. D65, 023521 (2001).

6. D. Lovelock, J. Math. Phys. 12, 498 (1971).

7. A. Balcerzak and M.P. Dąbrowski, Phys. Rev. D77, 023524 (2008).

8. M. Sasaki, T. Shiromizu and K.I. Maeda, Phys. Rev. D62, 024008 (2000); T. Shiromizu, K.I. Maeda, and M. Sasaki, Phys. Rev. D62, 024012 (2000); S. Mukohyama, T. Shiromizu, and K.I. Maeda, Phys. Rev. D62, 024028 (2000).

9. W. Israel, Nuovo Cimento B44, 1 (1966).

10. K.A. Meissner and M. Olechowski, Phys. Rev. Lett. 86, 3708 (2001).

11. N. Deruelle and T. Doležel, Phys. Rev. D62, 103502 (2000).

12. A. Jakubiec and J. Kijowski, Phys. Rev. D37, 1406 (1988).

13. H.H. v. Borzeszkowski and V.P. Frolov, Ann. Phys. (Leipzig) 7, 285 (1980).

14. M. Parry, S. Pichler, and D. Deeg, JCAP 0504, 014 (2005).

15. N. Deruelle, M. Sasaki, and Y. Sendouda, Prog.Theor.Phys., 119, 237 (2008).

16. G.W. Gibbons and S.W. Hawking, Phys. Rev. D15, 2752 (1977).

17. S.W. Hawking and J.C. Lutrell, Nucl. Phys. B247, 250 (1984).

18. M. Madsen and J.D. Barrow, Nucl. Phys. B323, 242 (1989).

19. T.S. Bunch, Journ. Phys. A14, L139 (1981).

20. F. Müller-Hoissen, Phys. Lett. B163, 106 (1985); R.C. Myers, Phys. Rev. D36, 392 (1987).

21. S.C. Davis, Phys. Rev. D67, 024030 (2003).

22. E. Gravanis and S. Willinson, Journ. Math. Phys. 47, 2503 (2006); Phys. Rev. D75, 084025 (2007).

23. A.D. Barvinsky and S.N. Solodukhin, Nucl. Phys. B479, 305 (1996).

24. A. Balcerzak and M.P. Dąbrowski, JCAP 01, 018 (2009).

25. V. Sahni, T.D. Saini, A.A. Starobinsky, and U. Alam, JETP Lett. 77, 201 (2003); M. Visser, Class. Quantum Grav. 21, 2603 (2004); R.R. Caldwell and M. Kamionkowski, JCAP 0409, 009 (2004); M.P. Dąbrowski and T. Stachowiak, Ann. Phys. (New York) 321, 771 (2006); M. Dunajski and G.W. Gibbons, Class. Quantum Grav. 25, 235012 (2008).

26. M.P. Dạbrowski, Phys. Lett. 625, 184 (2005); M.P. Da̧browski, Ann. Phys. (Berlin) 15, 352 (2006).

27. N.J. Poplawski, Phys. Lett. B640, 135 (2006); Class. Quantum. Grav. 24, 3013 (2007); S. Capozziello, V.F. Cardone, and V. Salzano, Phys. Rev. D78, 063504 (2008). 